\title{
CPR Guideline Chest Compression Depths May Exceed Requirements for Optimal Physiological Response
}

\author{
Olibhéar McAlister ${ }^{1,3}$, Daniel Guldenring ${ }^{2}$, Dewar D Finlay ${ }^{1}$, Raymond R Bond ${ }^{1}$, Hannah Torney ${ }^{1,3}$, \\ Ben McCartney ${ }^{3}$, Laura Davis ${ }^{3}$, Paul Crawford ${ }^{4}$, Adam Harvey ${ }^{3}$ \\ ${ }^{1}$ Ulster University, Newtownabbey, UK \\ ${ }^{2}$ University of Applied Sciences HTW Berlin, Berlin, Germany \\ ${ }^{3}$ HeartSine Technologies Ltd, Belfast, UK \\ ${ }^{4}$ Veterinary Anaesthesia Consultancy, Larne, UK
}

\begin{abstract}
A twelve-animal porcine study dataset was retrospectively analyzed to assess associations between chest compression (CC) depth, systolic blood pressure $(\mathrm{SBP})$ and end-tidal carbon dioxide $\left(\mathrm{EtCO}_{2}\right)$. Manual CCs were applied for 7 two-minute episodes, at CC depths between $10 \mathrm{~mm}-55 \mathrm{~mm}$. A rolling $15 \mathrm{~s}$ analysis window was applied to the continuous signals. Mean peak values were calculated for each window. Correlation analysis was applied to assess strength of association. Optimal CC depth to achieve physiological targets was determined via cut-off analysis.

A total of 672 observations for each variable were available for analysis. Pearson correlations $195 \%$ confidence interval; $p$-value) between CC depth and both $S B P$ and $E^{2} \mathrm{CO}_{2}$ were $0.84(0.82,0.86 ; p<0.001)$ and 0.75 (0.71, 0.78; $p<0.001)$ respectively. Optimal CC depth cutoff (sensitivity, specificity) to achieve $S B P \geq 100 \mathrm{mmHg}$ and $\mathrm{EtCO}_{2} \geq 10 \mathrm{mmHg}$ was $33 \mathrm{~mm}(98.29 \%, 88.94 \%)$ and 20 $\mathrm{mm}(95.08 \%, 78.30 \%)$ respectively.

$A$ reasonable relationship between $C C$ depth and physiological response was observed. Optimal SBP and $\mathrm{EtCO}_{2}$ cut-offs were achieved significantly below guideline depths. Furthermore, cut-off analysis suggests a disparity between CC depth and physiological targets.
\end{abstract}

\section{Introduction}

Cardiac arrest is a leading cause of premature death worldwide. To increase survival rates, early defibrillation and effective cardiopulmonary resuscitation (CPR) are crucial. American Heart Association (AHA) and European Resuscitation Council (ERC) basic life support guidelines consider chest compressions (CCs) at a rate of 100 to $120 \mathrm{CCs} \mathrm{min}^{-1}$ and a depth of 50 to $60 \mathrm{~mm}$ to be effective, amongst an adult population [1], [2].
Guideline CC depth between 50 and $60 \mathrm{~mm}$ has been proven to marginally increase survival to hospital admission, compared to previous guideline depths of 40 to $50 \mathrm{~mm}$ [3], [4]. While associated survival improves with deeper CCs so does the risk of causing injury to the patient [5]. It has been repeatedly reported that CPR performance is poor for both professional and lay rescuers, over several revisions of the basic life support guidelines [6], [7]. The low incidence of $\mathrm{CC}$ depth meeting the minimum guideline depth of $50 \mathrm{~mm}$ may be due to the target not being suitable for the entire adult population or early onset of fatigue [8], [9].

Research into patient response as an indicator of CPR quality is in its infancy with few physiological endpoints and cut-offs established. Advanced life support guidelines do suggest an alternative indicator of measuring $\mathrm{CC}$ quality. Observing a patient $\mathrm{EtCO}_{2}$ response of $<10 \mathrm{mmHg}$ is associated with mortality and efforts to improve CPR quality should be made. Supporting evidence suggests that continuous CCs between 100 and 120 compressions min $^{-1}$ maintains ideal blood pressure [10]. Friess et al. investigated the use of SBP as an indicator of CPR quality and used a physiological cut-off of $100 \mathrm{mmHg}$ [11].

\section{Methods}

The purpose of this analysis is to determine the optimal $\mathrm{CC}$ depth cut-off to achieve $\mathrm{EtCO}_{2} \geq 10 \mathrm{mmHg}$ and $\mathrm{SBP} \geq$ $100 \mathrm{mmHg}$. A retrospective analysis was conducted on a porcine dataset. The dataset included continuous timeseries data for $\mathrm{CC}$ depth, $\mathrm{EtCO}_{2}$ and SBP.

\subsection{Study Design}

All experiments were performed in accordance with the Home Office Guidance on the Operation of the Animals (Scientific Procedures) Act 1986 (UK).

Twelve $(\mathrm{n}=12)$ commercial pigs, aged approximately 
9 to 10 weeks and weighing between 30 to $35 \mathrm{~kg}$, were used in the study. Ventricular fibrillation (VF) was induced electrically, and the animals were left untreated for 3minutes. During the untreated period animals were ventilated at an approximate rate of 23 ventilations $\mathrm{min}^{-1}$.

Each animal had 7 episodes of continuous CCs applied at a rate of 110 compressions $\min ^{-1}$. The initial 4 episodes of CPR were applied to achieve an $\mathrm{EtCO}_{2}$ response of $<15 \mathrm{mmHg}$. The remaining 3 episodes targeted an $\mathrm{EtCO}_{2}$ response of $\geq 15 \mathrm{mmHg}$. There was a rest period of at least 10-seconds between CC episodes.

\subsection{Signal Data}

A HeartStart Mrx (Philips, USA) coupled with Q-CPR technology (Laerdal Medical, Norway) was used to record $\mathrm{CC}$ depth data. Depth signal data was captured at a sample frequency of $50 \mathrm{~Hz}$ and a resolution of $0.01 \mathrm{~mm}$ per least significant bit (LSB).

Physiological signals were recorded using a DatexOhmeda S/3 Anesthesia Monitor (GE Healthcare, USA) using VitalSignsCapture [12]. Side-stream capnograph was used to measure $\mathrm{EtCO}_{2}$ at a sampling frequency of $25 \mathrm{~Hz}$. Arterial blood pressure (BP) was captured from the carotid artery and sampled at a rate of $100 \mathrm{~Hz}$. Outputs from the anesthesia were recorded in $\mathrm{mmHg}$ and did not require scaling prior to processing.

\subsection{Data Processing}

An annotation review was conducted on the $\mathrm{CC}$ depth and BP signals, by study personnel, to identify the beginning of each CPR episode. The point of VF induction was also annotated on each BP signal. Annotated BP timepoints were transferable to the capnogram, as the same device was used to capture both signals. Episodes were segmented into 15-second epochs. An analysis widow was applied to each epoch to determine the amplitude of the signal.

Local minima were identified in the $\mathrm{CC}$ depth signal for each CC. The absolute value of the mean of the identified local minima, within a CC depth epoch, represented the mean $\mathrm{CC}$ depth.

Capnograph and BP signals were analyzed by peak envelope. The mean of the upper envelope in the capnograph and BP signals were taken as the representative values of $\mathrm{EtCO}_{2}$ and SBP for a given epoch, respectively.

Additional processing was applied to the capnograph signals. A rolling, non-overlapping, analysis widow was applied to each signal starting at the point of VF induction. The analysis window had a fixed duration of 12 -seconds which terminated after 180 -seconds of signal had been processed. The amplitude of the capnogram was calculated for each analysis window.

\subsection{Data Analyses}

Data was audited by independent review prior to analysis. $\mathrm{R}$ for statistical computing version 3.5.1 was used for all analyses.

Between-subject, within-subject and Pearson correlation analyses were applied to each combination of $\mathrm{CC}$ depth, $\mathrm{EtCO}_{2}$ and SBP [13], [14].

Cutoff analyses were applied to the data to determine the probabilistic $\mathrm{CC}$ depth cutoffs for $\mathrm{EtCO}_{2} \geq 10 \mathrm{mmHg}$ and peak BP $\geq 100 \mathrm{mmHg}$. Depth cutoffs increased in increments of $1 \mathrm{~mm}$ and accuracy, sensitivity, specificity and Youden index were calculated for each CC depth cutoff. Cutoffs which are associated with maximum accuracy and maximum Youden index were reported.

The decay of $\mathrm{EtCO}_{2}$ post $\mathrm{VF}$ induction was characterized by applying a log-log regression model to $\mathrm{EtCO}_{2}$ and time data.

\section{Results}

A total of 672 observations (12 animals $x 7$ episodes $\mathrm{x}$ 8 analysis windows) of $\mathrm{EtCO}_{2}$ and SBP were processed. There were 13 missing observations for CC depth due to the administration of shallow CCs.

There were non-significant, between-subject correlations observed for all combinations of CC depth, $\mathrm{EtCO}_{2}$ and SBP (Table 1).

Strong within-subject correlations were observed for all combinations of the study endpoints; $\mathrm{EtCO}_{2}$ and $\mathrm{CC}$ depth (0.83), $\mathrm{EtCO}_{2}$ and SBP (0.86) and SBP and CC depth (0.89). Additionally, lower, yet strong Pearson correlations were observed between all combinations of study endpoints. Further details of all correlation analyses are listed in Table 1.

The maximum accuracy cut-off for $\mathrm{CC}$ depth to predict $\mathrm{EtCO}_{2} \geq 10 \mathrm{mmHg}$ was $20 \mathrm{~mm}$. This provided an accuracy of $89.68 \%$ (sensitivity $=0.95$; specificity $=0.78$; Youden index $=0.73$ ). The optimal depth cut-off to classify $\mathrm{SBP} \geq 100 \mathrm{mmHg}$ was $33 \mathrm{~mm}$ with an accuracy of $92.26 \%$ (sensitivity $=0.98 ;$ specificity $=0.89 ;$ Youden Index $=0.87$ ).

Adjusting this analysis in favor of maximum Youden index the optimal cut-off for $\mathrm{EtCO}_{2} \geq 10 \mathrm{mmHg}$ increases to $21 \mathrm{~mm}$ with an accuracy of $87.86 \%$ (sensitivity $=0.90$; specificity $=0.84$; Youden index $=0.74)$. There was no change to the depth-cut off after adjusting for maximum Youden index.

The deterioration of $\mathrm{EtCO}_{2}$ post $\mathrm{VF}$ induction resembled characteristics indicative of exponential decay. Data obtained for each 12-second analysis window during the untreated duration of VF were not considered to be 
Table 1 Pearson, between-subject and within-subject correlation analyses for each combination of $\mathrm{EtCO}_{2}(\mathrm{n}$ $=672, \operatorname{SBP}(n=672)$ and CC depth $(n=659)$.

\begin{tabular}{|c|c|c|c|}
\hline Covariates & $\mathbf{r}$ & $95 \% \mathrm{CI}$ & p-value \\
\hline \multicolumn{4}{|l|}{ Between-Subjects } \\
\hline $\mathrm{EtCO}_{2}$ and Depth & -0.28 & $(-0.77,0.43)$ & 0.386 \\
\hline SBP and Depth & -0.22 & $(-0.75,0.48)$ & 0.491 \\
\hline $\mathrm{SBP}$ and $\mathrm{EtCO}_{2}$ & 0.35 & $(-0.35,0.80)$ & 0.258 \\
\hline \multicolumn{4}{|l|}{ Within-Subjects } \\
\hline $\mathrm{EtCO}_{2}$ and Depth & 0.83 & $(0.80,0.85)$ & $<0.001$ \\
\hline SBP and Depth & 0.89 & $(0.87,0.91)$ & $<0.001$ \\
\hline $\mathrm{SBP}$ and $\mathrm{EtCO}_{2}$ & 0.86 & $(0.84,0.88)$ & $<0.001$ \\
\hline \multicolumn{4}{|l|}{ Pearson } \\
\hline $\mathrm{EtCO}_{2}$ and Depth & 0.75 & $(0.71,0.78)$ & $<0.001$ \\
\hline SBP and Depth & 0.84 & $(0.82,0.86)$ & $<0.001$ \\
\hline $\mathrm{SBP}$ and $\mathrm{EtCO}_{2}$ & 0.80 & $(0.78,0.83)$ & $<0.001$ \\
\hline
\end{tabular}

normally distributed (Figure 1). The median value for each timepoint was applied to a regression model to trend the decay. A log-log model was trained after applying a natural $\log$ transform to both $\mathrm{EtCO}_{2}$ and time data. This provides the following model satisfied in terms of $\operatorname{EtCO}_{2}(y)$ as a response of time $(x)$ :

$$
y=e^{-0.71 \cdot \log _{e}(x)+5.45}
$$

The coefficient of determination for the model $\left(\mathrm{R}^{2}\right)$ was 0.996, suggesting the model is a good fit for the data.

\section{Discussion}

The analysis conducted could not identify a relationship between animals which received higher $\mathrm{CC}$ depths and those which produced higher $\mathrm{EtCO}_{2}$ or SBP responses. This may be consequential to the damage which occurred to one animal's thorax or that each animal received the same treatment.

The Pearson correlation analysis suggests there is a moderate relationship between $\mathrm{CC}$ depth and $\mathrm{EtCO}_{2}$ (0.75). Comparisons of CC depth and SBP show a stronger relationship (0.84). Comparing the Pearson and withinsubject correlation analyses, however, suggests there is an improvement in the relationship, between the covariates, when fitting for each animal.

The binary outcome for $\mathrm{EtCO}_{2} \geq 10 \mathrm{mmHg}$ was predicted accurately using a CC depth cut-off of $20 \mathrm{~mm}$; Accuracy (89.68\%) and Youden index (0.73). Similarly, binary outcome for SBP was accurately predicted with a depth cut-off of $33 \mathrm{~mm}$; Accuracy (92.26\%) and Youden index (0.87).

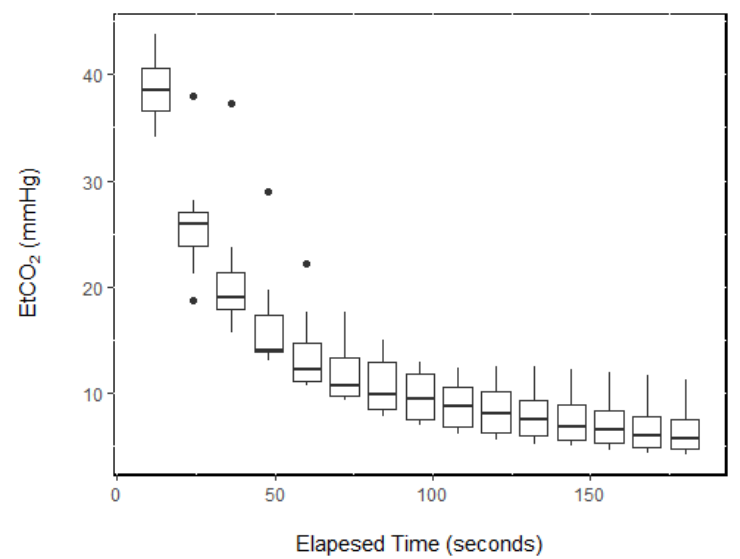

Figure 1 Boxplot series displaying the exponential decay of $\mathrm{EtCO}_{2}$ as function of time, after induction of VF. $(\mathrm{N}=144 ; 12$ observations per time point $\mathrm{x}$ 12 timepoints)

Depths required to meet the physiological targets however, are considerably lower than the current guideline recommended depths of 50 to $60 \mathrm{~mm}$. It is also worth noting that depths required to achieve the defined physiological cut-offs were also not in agreeance. This reflects the conclusion of Steill et al, that an increase in CC depths is associated with better patient outcomes, however, the optimal depth of CC is still unknown [9].

One animal suffered extensive thoracic trauma as a result of receiving CC depths in excess of $45 \mathrm{~mm}$. Complications due to this treatment included bleeding into the thorax, bleeding into the pericardial sac, bruised myocardium, bullae on the lungs and most severely ruptured atria. This highlights that a balanced approach to delivering safe and efficacious CC depth targets is required. This study demonstrated depths greater than $45 \mathrm{~mm}$ were acceptable for 11 of the 12 enrolled animals, however, one displayed irreversible damage as a result of this treatment. At what point are the CC depth targets considered safe for use on the human population?

Upon visual inspection of the synchronized signals, it is apparent that $\mathrm{EtCO}_{2}$ is a slow response variable, which does not reflect sudden changes in applied CC depth

\section{Limitations}

The investigation was a retrospective analysis of a previously obtained dataset. The objective of the original study did not match the objective of this post hoc analysis. Treatment was not randomized during the study. Depth of CC increased with each episode which may have an indirect impact on physiological response of the animal. As observed as part of the analysis, $\mathrm{EtCO}_{2}$ had a decay artefact and requires a considerable amount of time to baseline, perhaps the 10-second interval between CC episodes would need extended to accommodate this. 

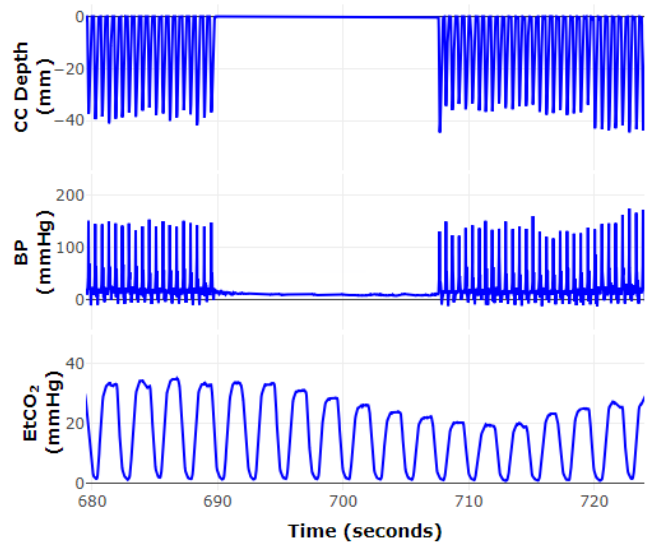

Figure 2 Representative time series plots of CC depth, $\mathrm{BP}$ and $\mathrm{EtCO}_{2}$, demonstrating the slow response of $\mathrm{EtCO}_{2}$ to sudden changes in $\mathrm{CC}$ depth

\section{Conclusions}

This investigation provides encouraging preliminary results indicating that $\mathrm{CC}$ depths recommended by AHA and ERC guidelines may be excessive. As this is a retrospective analysis further research is required to establish the relationship between animals for $\mathrm{CC}$ depth and the physiological endpoints of $\mathrm{EtCO}_{2}$ and $\mathrm{BP}$.

\section{Conflicts of Interest}

Olibhéar McAlister, Hannah Torney, Ben McCartney, Laura Davis and Adam Harvey are employees of HeartSine Technologies Ltd. Paul Crawford is a consultant veterinary anesthetist contracted by HeartSine Technologies Ltd.

\section{Acknowledgements}

The authors of this paper would like to thank the staff at the Roslin Institute (The University of Edinburgh) for their help collecting the presented data.

\section{References}

[1] M. E. Kleinman et al., "Part 5: Adult basic life support and cardiopulmonary resuscitation quality: 2015 American Heart Association guidelines update for cardiopulmonary resuscitation and emergency cardiovascular care," Circulation, vol. 132, no. 18, pp. S414-S435, 2015.
[2] J. Soar et al., "European Resuscitation Council Guidelines for Resuscitation 2015. Section 3. Adult advanced life support.," Resuscitation, vol. 95, pp. 100$147,2015$.

[3] T. Vadeboncoeur et al., "Chest compression depth and survival in out-of-hospital cardiac arrest," Resuscitation, vol. 85, no. 2, pp. 182-188, Feb. 2014.

[4] C. H. Wang et al., "Outcomes of adults with in-hospital cardiac arrest after implementation of the 2010 resuscitation guidelines," Int. J. Cardiol., vol. 249, pp. 214-219, 2017.

[5] H. Hellevuo et al., "Deeper chest compression - More complications for cardiac arrest patients?," Resuscitation, vol. 84, no. 6, pp. 760-765, Jun. 2013.

[6] B. S. Abella, "Quality of Cardiopulmonary Resuscitation During In-Hospital Cardiac Arrest," Jama, vol. 293, no. 3, p. 305, Jan. 2005.

[7] T. Gyllenborg, A. Granfeldt, F. Lippert, I. S. Riddervold, and F. Folke, "Quality of bystander cardiopulmonary resuscitation during real-life out-of-hospital cardiac arrest," Resuscitation, vol. 120, pp. 63-70, Nov. 2017.

[8] T.-G. Kampmeier et al., "Chest compression depth after change in CPR guidelines-Improved but not sufficient," Resuscitation, vol. 85, no. 4, pp. 503-508, Apr. 2014.

[9] I. G. Stiell et al., "What Is the Optimal Chest Compression Depth During Out-of-Hospital Cardiac Arrest Resuscitation of Adult Patients?," Circulation, vol. 130, no. 22, pp. 1962-1970, Nov. 2014.

[10] L. M. Cunningham, A. Mattu, R. E. O'Connor, and W. J. Brady, "Cardiopulmonary resuscitation for cardiac arrest: the importance of uninterrupted chest compressions in cardiac arrest resuscitation," Am. J. Emerg. Med., vol. 30, no. 8, pp. 1630-1638, Oct. 2012.

[11] S. H. Friess et al., "Hemodynamic directed CPR improves cerebral perfusion pressure and brain tissue oxygenation," Resuscitation, vol. 85, no. 9, pp. 1298 1303, Sep. 2014.

[12] J. Karippacheril and T. Ho, "Data acquisition from $\mathrm{S} / 5$ GE Datex anesthesia monitor using VSCapture: An open source.NET/Mono tool," J. Anaesthesiol. Clin. Pharmacol., vol. 29, no. 3, p. 423, 2013.

[13] J. M. Bland and D. G. Altman, "Statistics notes: Calculating correlation coefficients with repeated observations: Part 1--correlation within subjects," BMJ, vol. 310, no. 6977, pp. 446-446, Feb. 1995.

[14] J. M. Bland and D. G. Altman, "Statistics notes: Calculating correlation coefficients with repeated observations: Part 2--correlation between subjects," $B M J$, vol. 310, no. 6980, pp. 633-633, Mar. 1995.

Address for correspondence.

Olibhéar McAlister

Ulster University, NIBEC, Newtownabbey, BT37 OQB

McAlister-o2@ulster.ac.uk 